\title{
Aprendiendo geometría de curvas planas con MATLAB
}

\section{Fernando Giménez ${ }^{\mathrm{a}}$, Juan A. Monsoriu ${ }^{\mathrm{b}}$, Antonio J. Jiménez ${ }^{\mathrm{c}}$ y Andres Lapuebla ${ }^{\mathrm{d}}$}

aDepartamento de Matemática Aplicada, Universitat Politècnica de València, Spain, fgimenez@mat.upv.es, bDepartamento de Física Aplicada, Universitat Politècnica de València, Spain, jmonsori@fis.upv.es, 'Dpto. de Mecánica de los Medios Continuos y Teoría de Estructuras, Universitat Politècnica de València, Spain, ajimenez@mes.upv.es, ${ }^{\mathrm{d}}$ Dpto. de Mecánica de los Medios Continuos y Teoría de Estructuras, Universitat Politècnica de València, Spain, anlafer0@mes.upv.es.

\begin{abstract}
The study of geometric properties of plane curves has a great interest in several technical fields. It has many applications in Civil and Industrial Engineering, Architecture, Physics, etc. Displaying such properties for any type of curve allows technicians - and especially students of several technical degrees - to consolidate their knowledge on the subject. In this work we develop a Matlab-based graphical application that allows the visualization of geometric properties of plane curves in an interactive ways. It also permit the encouragement and independent learning of students.
\end{abstract}

Keywords: Virtual laboratori, Matlab, plane curve, tangent vector, normal vector,curvature, center of curvature, osculating circle, involute

\section{Resumen}

El estudio de las propiedades geométricas de las curvas planas es de gran interés en muchos campos de las ingenierías de caminos, industrial, arquitectura, de la física, etc. y presentan múltiples e importantes aplicaciones. La visualización de dichas propiedades para cualquier tipo de curva permite que los alumnos puedan afianzar sus conocimientos sobre el tema. En este trabajo desarrollamos una aplicación de Matlab que permite dicha visualización de manera interactiva y que fomenta el aprendizaje autónomo de los alumnos.

Palabras clave: Laboratorio virtual, Matlab, curva plana, vector tangente, vector normal,curvatura, centro de curvatura, círcunferencia osculatriz, evoluta 


\section{Introducción}

El uso de las nuevas tecnologías dentro del proceso de enseñanza-aprendizaje ha ido adquiriendo durante los últimos años cada vez más importancia, lo que ha permitido que los alumnos pueden adquirir más fácilmente competencias y capacidades (Benito, 2006). En este sentido se debe de destacar las posibilidades que ofrece el uso de del ordenador, con sus enormes capacidades de cálculo y gráficas, en la enseñanza de asignaturas de carácter técnico y científico, facilitando la transmisión de conocimientos. La utilización por parte de los alumnos de los laboratorios virtuales permiten trabajar eficientemente muchos conceptos de los campos de las matemáticas, física, ingeniería, etc. de manera que el estudiante adquiere un papel activo en el proceso de enseñanza-aprendizaje (Depcik, 2005).

En este trabajo presentamos una Interfaz Gráfica de Usuario (GUI) de Matlab que puede utilizarse en las prácticas docentes de aquellas asignaturas universitarias que estudien la teoría de curvas planas. El laboratorio virtual permite visualizar de forma dinámica las propiedades geométricas de una curva plana tales como la curvatura, la longitud de arco, los vectores tangentes y normales, el círculo osculador, la evoluta, etc. Pueden consultarse las referencias (Barragán, 2014) y (Mathworks, 2015) sobre las GUI de Matlab.

Otros trabajos relacionados con el uso de laboratorios virtuales y herramientas informáticas para el estudio de curvas son los de GIEMATIC, (Benitez, 2012), (Mora, 2012) y (Cuadrado, 2015).

\section{Objetivos}

Con la herramienta que hemos desarrollado se intenta afianzar los conocimientos teóricos adquiridos por los alumnos sobre la teoría de curvas en el plano, dedicando una especial atención a:

- Visualización de los vectores tangentes y normales a una curva del plano generada a partir de su expresión en coordenadas cartesianas, paramétricas o polares.

- Establecer diferencias entre las distintas parametrizaciones que puede tener una curva.

- Interpretación de una curva en paramétricas y sus propiedades a partir del movimiento de un punto en un plano.

- Conocimiento del concepto de longitud de arco y valoración de su importancia en la geometría plana (parametrización).

- Obtención e interpretación geométrica de la curvatura a lo largo de los puntos de la curva.

- Estudio del concepto de ángulo de giro y su relación con la curvatura.

(cc) EY-Nc-ND 2015, Universitat Politècnica de València 
- Conocimiento y visualización de los conceptos de centro de curvatura, círcunferencia osculatriz, evoluta y su relación con la curvatura.

- Estudio de los distintos tipos de coordenadas más usados para la definición de curvas.

- Estudio de alguna de las curvas planas más famosas.

\section{Desarrollo de la innovación}

En el trabajo se presenta un laboratorio virtual enfocado al estudio de las propiedades geométricas de las curvas planas por parte de los alumnos de ingeniería y arquitectura. Se comienza introduciendo los conceptos de vector tangente, vector normal, longitud de arco, ángulo de giro, curvatura, centro de curvatura, círcunferencia osculatriz, evoluta, etc. y se dan las expresiones que permiten su cálculo. A continuación se presenta detalladamente la interfaz gráfica de usuario de Matlab. Por medio de ejemplos se muestra en tiempo real información gráfica y analítica de cada uno de los distintos conceptos que se han estudiado previamente en función del parámetro que define la curva, de manera que el alumno pueda afianzarlos y comprenderlos mejor.

Una de las características de la aplicación es que se obtiene información relevante sobre las curvas planas a partir de gráficos animados para cada uno de los conceptos que se estudian, de forma que se aprecia de forma dinámica la variación de las propiedades geométricas a lo largo de los distintos puntos de la curva, en función de los valores del parámetro que la define.

Se ha diseñado esta herramienta para su uso tanto en las aulas informáticas como en ordenadores particulares, aunque no se disponga del paquete Matlab, ya que se ha generado una versión ejecutable de la misma. En cualquier caso se facilitará al alumno una guía detallada del laboratorio virtual con una descripción de los distintos parámetros de la aplicación y acompañada de ejemplos ilustrativos.

\subsection{Teoría de curvas en el plano}

Una curva puede considerarse como una línea continua de una dimensión, que varía de dirección paulatinamente. En el caso de curvas planas muchas de ellas se pueden obtener como la traza $\alpha(I)$ de una aplicación $\alpha: I \rightarrow \mathbb{R}^{2}$, donde $I$ es in intervalo. Se dice que la curva $C=\alpha(I)$ está parametrizada por $\alpha$. 
Si $\alpha$ es diferenciable se llama vector tangente o vector velocidad a $\alpha^{\prime}(t)$. El vector tangente unitario es $T=\frac{\alpha^{\prime}(t)}{\left\|\alpha^{\prime}(t)\right\|}$ si $\alpha^{\prime}(t) \neq 0$. Si $J((u, v))=(-v, u)$ a $N=J(T)$ se le llama vector normal unitario.

La función longitud de arco $s: I \rightarrow \mathbb{R}^{2}$ se define por $s(t)=\int_{a}^{t}\left\|\alpha^{\prime}(u)\right\| d u$ donde $a$ es el extremo izquierdo de $I$.

La curvatura de $\alpha$ en el punto $t$ se define por

$$
k(t)=\frac{\alpha^{\prime}(t) \cdot J \alpha^{\prime}(t)}{\left\|\alpha^{\prime}(t)\right\|^{3}}
$$

Se sabe que la curvatura de una curva mide la desviación de ésta respecto a una dirección prefijada. Es una medida de cómo cambia el campo de vectores tangentes.

El ángulo de giro $\beta(t)$ de $\alpha$ en el punto $t$ se define como el ángulo comprendido entre el vector tangente y el eje de abcisas.

El círculo osculador a una curva en un punto dado es una circunferencia cuyo centro se encuentra sobre la recta normal a la curva y tiene la misma curvatura que la curva dada en ese punto. Al centro y el radio de este círculo se les concoce como centro de curvatura y radio de curvatura respectivamente. El radio de curvatura solo está definido si $\alpha^{\prime}(t) \neq 0$ y coincide $\operatorname{con} \frac{1}{k(t)}$.

Se llama evoluta al lugar geométrico de los centros de curvatura.

Para profundizar sobre la geometria de curvas se puden consultar las referencias (Roman, 2013), (Puente, 2007) y (Lastra, 2015).

\subsection{El laboratorio virtual}

La aplicación curvaplana que presentamos puede verse en la figura 1. Debajo del título se encuentran los campos donde el usuario introduce los parámetros de entrada.

Los parámetros de entrada son:

- Tipo de coordenadas: paramétricas, cartesianas y polares

- Expresión de la curva: campo en donde el usuario debe de introducir la expresión que define la curva en las diferentes coordenadas. En el caso de coordenadas paramétricas o polares el parámetro tiene que ser forzosamente $t$. En el caso de coordenadas cartesianas tiene que ser $x$.

- Valor inicial: corresponde al valor inicial del parámetro t o x según el caso. 
- Tipo de gráfica: curva, longitud de arco, curvatura, ángulo de giro y círculo osculador.

En el caso de seleccionar curva se genera un gráfico animado de los vectores tangente y normal a lo largo de la curva.

En el caso de seleccionar círculo osculador se genera un gráfico animado de un punto que se va moviendo a lo largo de la curva en función del parámetro que la define, el círculo correspondiente círculo osculador y la evoluta.

En el resto de casos se generan dos gráficos animados: el primero muestra un punto que se va moviendo a lo largo de la curva en función del parámetro que la define y el segundo a un punto que se va moviendo a lo largo de la representación gráfica de la longitud de arco, curvatura y ángulo de giro según el caso.

El botón Pulsar botón procede a la generación de los gráficos.

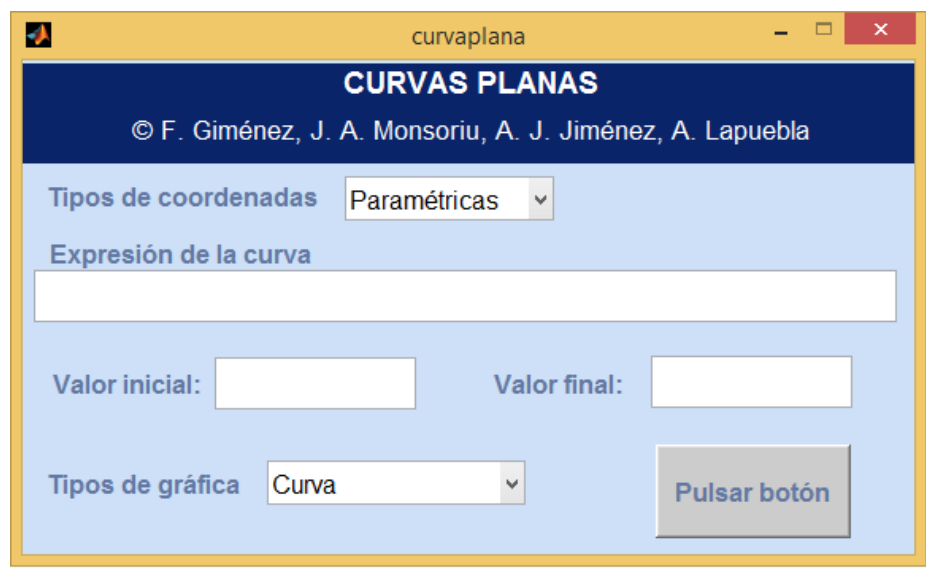

Fig. 1 Interfaz del laboratorio virtual

\subsubsection{Ejemplo}

En este ejemplo se muestra una captura correspondiente a la curva

$$
\begin{aligned}
& x=4 \cos (t)+\cos (4 t) \\
& y=4 \operatorname{sen}(t)-\operatorname{sen}(4 t)
\end{aligned}
$$

para $t \in[0,2 \pi]$ (hipocicloide) y a los vectores tangente y normal en $t=1.8405$.

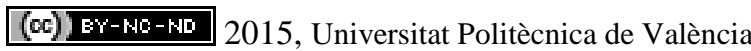


Aprendiendo geometría de curvas planas con MATLAB

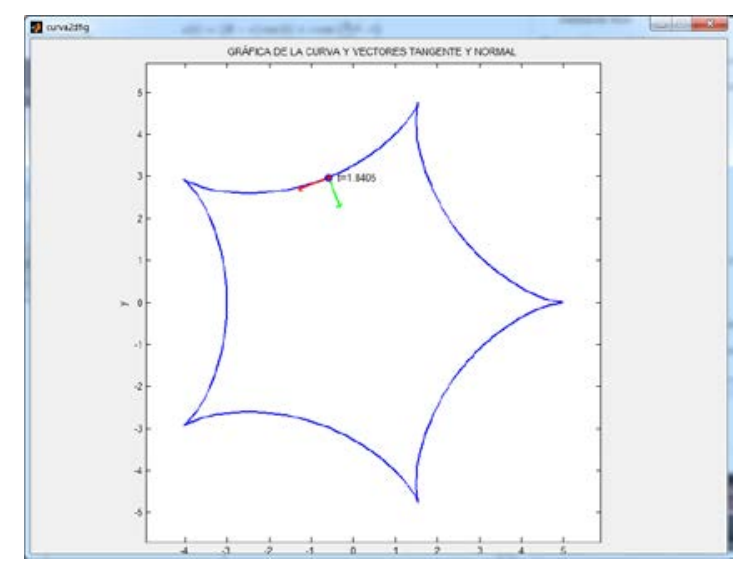

Fig. 2. Gráfica de la curva hipocicloide del ejemplo 3.2.1 y vectores tangente (rojo) y normal (verde).

\subsubsection{Ejemplo}

En la siguiente figura se muestra las gráficas de la curva $y=x \cos \left(x^{2}\right), x \in[0,3]$ y su longitud de arco. Se puede apreciar que la función longitud de arco no es lineal en este caso. Puede observarse como la longitud de arco adquiere mayor pendiente en los puntos donde crece o decrece más rápidamente la curva.

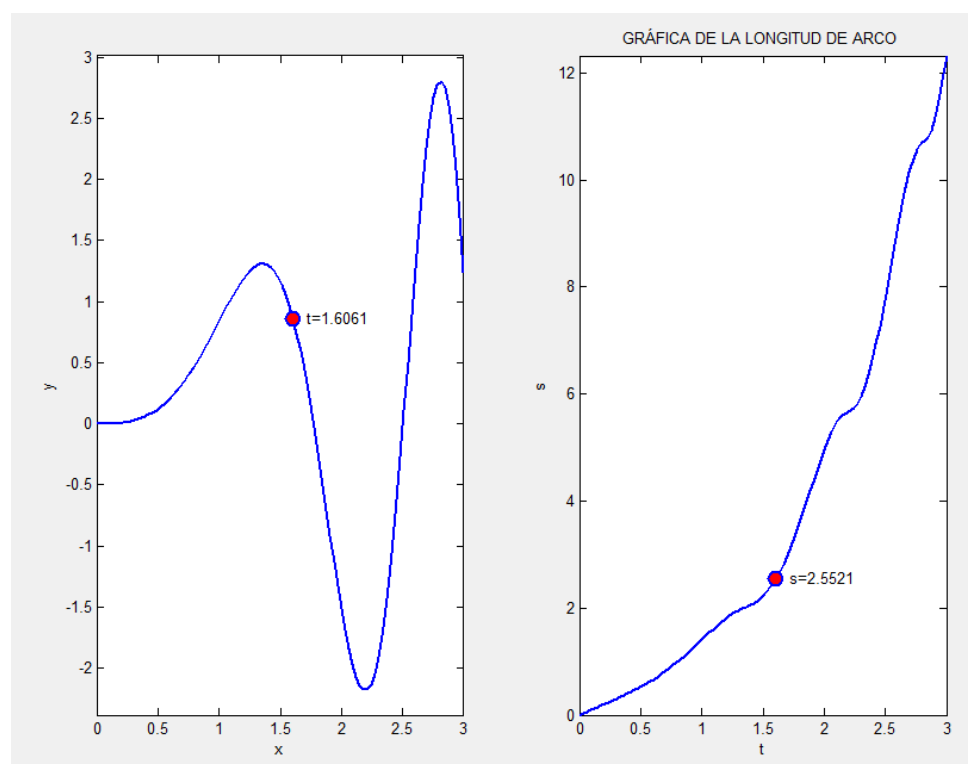

Fig. 3. Gráfica de la curva del ejemplo 3.2.2 y su longitud de arco

(cc) EY-NC-ND 2015, Universitat Politècnica de València

Congreso In-Red (2015) 


\subsubsection{Ejemplo}

Las siguientes tres figuras presentan las gráficas de la curvatura, el ángulo de giro y varias capturas del círculo osculador correspondientes a diversos valores del ángulo de la curva en polares $r=1+\cos (t), t \in[0,2 \pi]$ (cardioide).

En la figura 4 se observa que en el punto $(0,2)$ de la curva (el correspondiente a $t=0$ ) la curvatura es $0 \mathrm{y}$ en el punto $(0,0)$ (el correspondiente a $t=\pi$ ) la curvatura se hace infinita. También se aprecia en la figura 6 que la evoluta de la cicloide es otra cicloide más pequeña y orientada en sentido inverso.

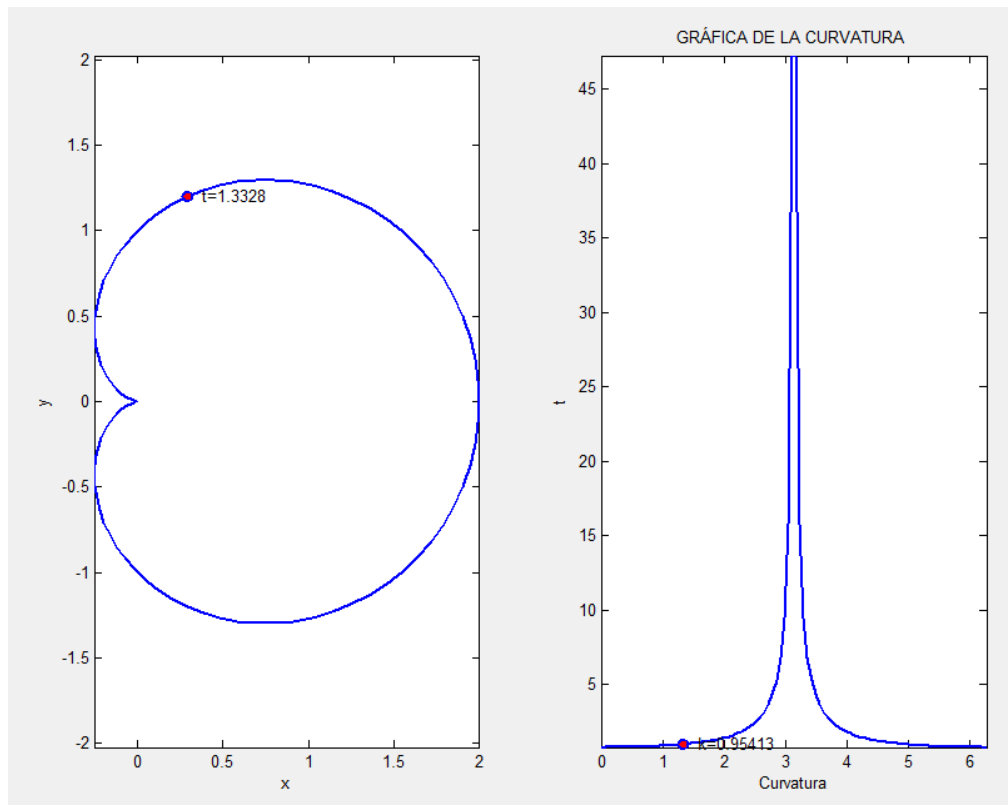

Fig. 4. Gráfica de la cardioide y su curvatura 
Aprendiendo geometría de curvas planas con MATLAB
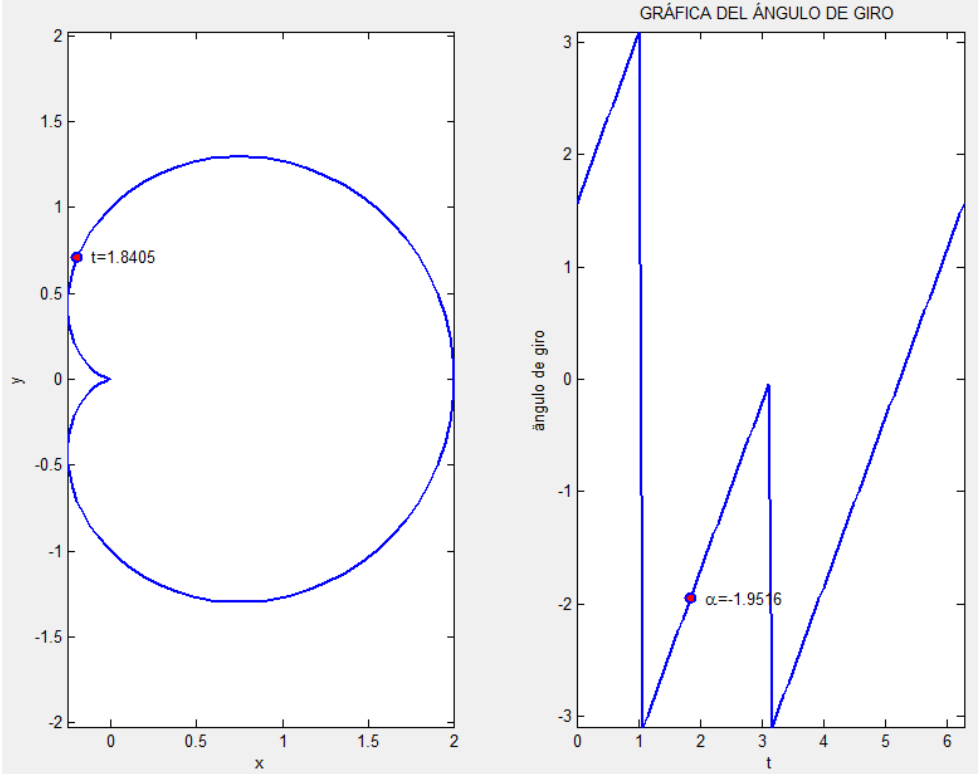

Fig. 5. Gráfica de la cardioide y su ángulo de giro
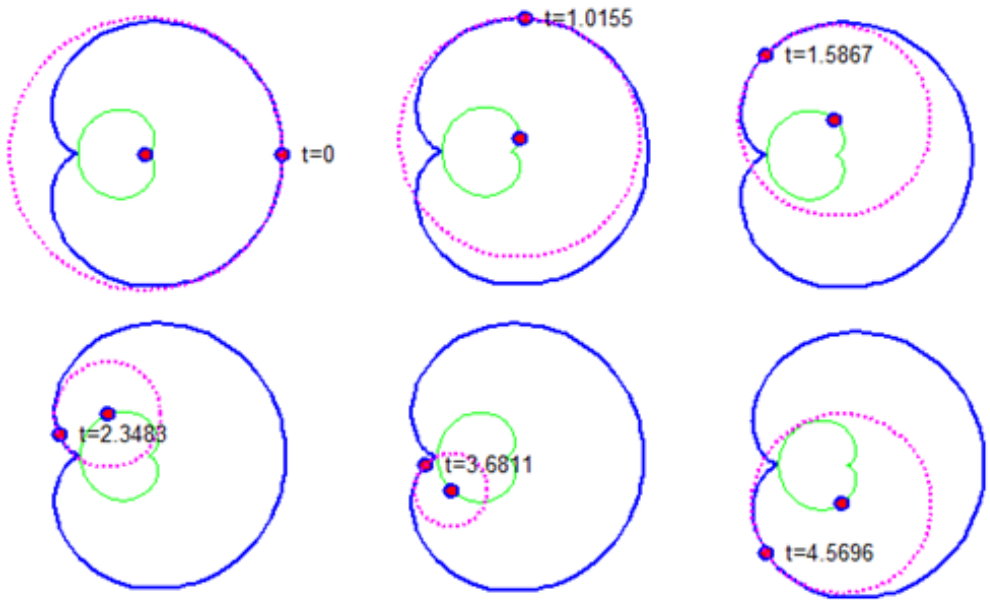

Fig. 6. Gráficas del círculo osculador y la evoluta de la cardioide para diversos valores de t 


\section{Resultados}

Con está herramienta creemos que el alumno podrá asimilar más fácilmente los conceptos relacionados con la teoría de curvas planas, de manera que la información de tipo gráfico que aporta el laboratorio virtual ayude a la compresión de la parte teórica.

Se propone la siguiente metodología a seguir en las asignaturas de matemáticas de las ingenierías y, en general, en aquellas disciplinas de carácter científico en donde se necesita conocer y manejar la geometría diferencial de curvas:

1) Introducción en las clases habituales de aula de los conceptos teóricos necesarios.

2) En las clases de laboratorio de ordenador se explicará con detalle como se usa la herramienta docente para obtener información relativa a las curvas planas y sus propiedades.

3) Se propondrán una serie de ejercicios ilustrativos en donde los alumnos tengan que deducir a partir de la información gráfica proporcionada por la aplicación informática aspectos relacionados con las propiedades geométricas de las curvas. A continuación se trataría de que los estudiantes resuelvan algunos de loa problemas planteados de forma analítica.

Para valorar la incidencia de esta metodología se propone la realización de una pequeña encuesta al final de la sesión práctica.

Los pasos 2) y 3) propuestos también son susceptibles de realizarse de manera autónoma y autodidacta por parte del alumno en su propio ordenador, incluso aunque el alumno no disponga del paquete de software Matlab en su casa, mediante el uso de una versión ejecutable del laboratorio virtual que hemos desarrollado. Se facilitará al alumno una guía detallada del laboratorio virtual.

\section{Conclusiones}

El laboratorio virtual y la propuesta metodológica se ha diseñado para su utilización en aquellas asignaturas de matemáticas de los primeros cursos de ingeniería en donde se estudien en profundidad la teoría de curvas en el plano. La sencillez de manejo, rapidez, opciones gráficas e interactividad pueden ser útiles a la hora de profundizar sobre muchos de los conceptos geométricos que se estudian.

En un futuro se desea completar el trabajo desarrollando una aplicación informática para el estudio de curvas en el espacio, prestando especial atención a que el alumno comprenda el triedro de Frenet (vectores tangente, normal y binormal), curvatura, torsión, longitud de arco, círculo osculador, etc.

(cc)) EY-NC-ND 2015, Universitat Politècnica de València

Congreso In-Red (2015) 
Aprendiendo geometría de curvas planas con MATLAB

\section{Agradecimientos}

Los autores agradecen al Instituto de Ciencias de la Educación de la Universitat Politècnica de València por su ayuda al Equipo de Innovación y Calidad Educativa MOMA.

\section{Referencias}

BARRAGAN, D. La web de MATLAB, SIMULINK, VHDL, microcontroladores... $<$ http://www.matpic.com> [Consulta: 10 de mayo de 2015]

BENITEZ, J. (2008) Curvas rectificables. Valencia: Universitat Politècnica de València, $<$ https://riunet.upv.es/handle/10251/2021>. [Consulta: 10 de mayo de 2015]

BENITO, A., PORTELA, A. y RODRÍGUEZ, R. M. (2006). “Análisis de la enseñanza de la Física en Europa: el fomento de competencias generales en estudiantes universitario”. Revista Iberoamericana de Educación, número 38/7.

CUADRADO, J. A. (2015) Aplicaciones educativas para díbujo técnico. <http://jcuadra2.wix.com/cuadrado\#!dibujo-tcnico/c1rvq>. [Consulta: 10 de mayo de 2015]

DEPCIK, C. y ASSANIS, D.N. (2005). "Graphical user interfaces in an engineer in educational environment”. Comput. Appl. Eng. Educ. Vol. 13.

GIEMATIC UC (2012)

<http://www.giematic.unican.es/variasVariables/laboratorios/CurvasSuperficies/indexgie.html> [Consulta: 10 de mayo de 2015]

LASTRA, A. (2015) Geometría de curvas y superficies con aplicaciones en arquitectura. Ed. Paraninfo.

MATHWORKS, (2015) MATLAB® Creating Graphical User Interfaces. The MathWorks, Inc. MORA, J: A. (2012) Taller: Rectas y curvas de colores.

$<$ http://jmora7.com/Color/index.htm>. [Consulta: 10 de mayo de 2015]

PUENTE, M. J. (2007) Curvas algebraicas y planas. Servicio Publicaciones Universidad de Cadiz ROMAN, J. de B. (2013) Curvas planas y en el espacio. García Maroto Editores.

ZOIDO, R. J. (2008) "Curvas y superficies en la arquitectura".Segundo Congreso Internacional de Matemáticas en la Ingeniería y la Arquitectura. 